\title{
Role of elasto-plastic analysis under cyclic loading in fatigue crack growth studies
}

\author{
B DATTAGURU
}

Department of Aerospace Engineering, Indian Institute of Science, Bangalore 560012, India

e-mail: datgur@aero.iisc.ernet.in

\begin{abstract}
Linear Elastic Fracture Mechanics (LEFM) has been widely usedin the past for fatigue crack growth studies, but this is acceptable only in situations which are within small scale yielding (SSY). In many practical structural components, conditions of SSY could be violated and one has to look for fracture criteria based on elasto-plastic analysis. Crack closure phenomenon, one of the most striking discoveries based on inelastic deformations during crack growth, has significant effect on fatigue crack growth rate. Numerical simulation of this phenomenon is computationally intensive and involved but has been successfully implemented. Stress intensity factors and strain energy release rates lose their meaning, $J$-integral (or its incremental) values are applicable only in specific situations, whereas alternate path independent integrals have been proposed in the literature for use with elasto-plastic fracture mechanics (EPFM) based criteria. This paper presents certain salient features of two independent finite element (numerical) studies of relevance to fatigue crack growth, where elasto-plastic analysis becomes significant. These problems can only be handled in the current day computational environment, and would have been only a dream just a few years ago.
\end{abstract}

Keywords. Fatigue crack growth; material nonlinearity; finite element analysis.

\section{Introduction}

Fracture mechanics based design has become mandatory for crucial structural components in high technology industries. The current technological viewpoint is design based on damage tolerance and to aim to specify life till the damage grows to unacceptable levels. The field is entirely inter-disciplinary, requiring inputs from both experimental and numerical methods. Experimental methods required include accurate methods for elastic and inelastic material property evaluation, load estimation in-service, non-destructive testing (NDT) and full scale fatigue testing. Computational methods in structural mechanics such as 
finite element or boundary element methods provide stress analysis of cracked bodies and post-process fracture parameters such as stress intensity factors or strain energy release rates. Data from both these are fed as input for analysis of failure or fatigue crack growth for life estimation/extension. For a successful conduct of this program, it is necessary to use advanced numerical methods to analyse the structure with realistic assumptions covering large deformations, elasto-plastic, visco-elastic/plastic material behaviour. This paper focuses attention on elasto-plastic material behaviour and its role in fatigue crack growth studies in metallic structures under cyclic loading.

In many problems, involving small-scale yielding (SSY) at the crack tip, linear elastic fracture mechanics (LEFM) has been used in the past for fatigue crack growth studies and life estimation. Several well-known models are in practice based on LEFM to estimate fatigue crack growth using numerical estimates of stress intensity factors or strain energy release rates (or $J$ ) (Broek 1978). In many practical structures and, in particular, structural components such as those used in aerospace vehicles, SSY could be violated and one has to look for fracture criteria based on elasto-plastic analysis.

The crack closure phenomenon proposed by Elber (1970) was one of the most striking discoveries which modified fatigue crack growth expressions in terms of an effective stress intensity range. Practical uses of this phenomenon are generally limited by the difficulties in experimental measurements and complexity in numerical simulation to estimate crack closure stress and effective stress intensity range. Recently, success is reported in experimental measurements (Sunder \& Dash 1982) and in numerical simulation, though the problem is still computationally intensive. Even in the absence of crack closure, fatigue crack growth analysis has complications. Certain fracture parameters are not valid under cyclic loading (or in case of loading/unloading). Under elasto-plastic deformation and cyclic loading, stress intensity factors and strain energy release rates lose their meaning, $J$-integral (or its incremental) values are applicable only in the case of deformation theory of plasticity (Rice 1968). Due to this, alternate path independent integrals were proposed by Atluri \& Nishioka (1982) for use in these cases.

This paper presents two different finite element studies of relevance to fatigue crack growth. The first deals with the numerical simulation of crack closure phenomenon and its use in study of the effect of low-high and high-low blocks of constant amplitude loading on a standard compact tension (CT) specimen. The major issues related to the numerical simulation are highlighted. In the second, fatigue crack growth around the interference lug joints is analysed in the presence of considerable inelastic deformation around the joint. Here $\Delta T_{p}^{*}$ integrals proposed by Atluri \& Nishioka (1982) were used to fit the fatigue crack growth data with success.

\section{Literature}

It is physically impossible to review the entire literature pertaining to elasto-plastic finite element analysis. A brief review of literature is presented here pertaining to fracture related work dealing with elasto-plastic analysis under cyclic loading keeping our focus on the theme of this paper. 


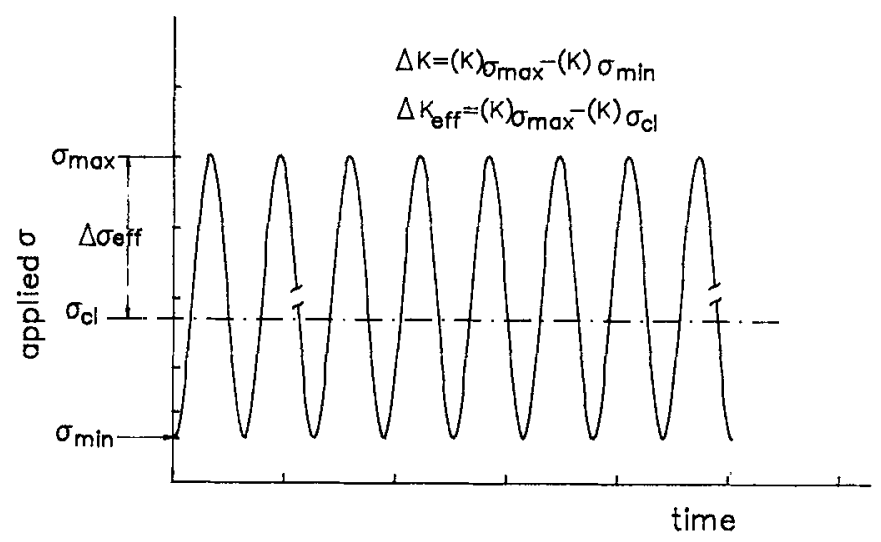

Figure 1. Constant amplitude loading on a cracked plate.

\subsection{Fatigue crack growth}

In general, loads on a practical structure are cyclic and vary in magnitude. The loading is represented by a maximum and a minimum applied stress/load in each cycle. If the maximum and minimum vary from cycle to cycle, the loading is referred to as variable amplitude loading, and if they are constant then the loading is constant amplitude (CA) loading.

The difference between the stress intensity factor values between the maximum and the minimum stress is known as stress intensity range $\Delta K$ (figure 1). It was Paris et al (1961) who examined a large body of experimental data and gave an empirical relation between stress intensity factor range $(\Delta K)$ and crack growth rate $(\mathrm{d} a / \mathrm{d} n)$. They proposed a relation

$$
\mathrm{d} a / \mathrm{d} n=C(\Delta K)^{m},
$$

where $C$ and $m$ are material constants. When predictions on the basis of (1) are made, it is assumed that the only tensile part of the load cycle contributes to the fatigue damage and that crack tip surfaces close at zero load. Forman et al (1967) modified the Paris equation taking into account the stress ratio $R$ (ratio of minimum to maximum stress in a cycle).

The discovery of crack closure by Elber (1970) led to the modification of the Paris equation as

$$
\mathrm{d} a / \mathrm{d} n=C\left(\Delta K_{\text {eff }}\right)^{m},
$$

where $\Delta K_{\text {eff }}$ is the effective stress intensity range between the maximum stress and crack closure stress. He attributed crack closure to local material yielding near the crack tip and residual plastic wake behind the crack tip during the crack growth phase. This can cause the crack tip to close even under a positive applied stress.

\subsection{Elasto-plastic analysis}

Finite element analysis including material nonlinear behaviour is briefly described later in this paper. Nonlinear material characteristics are determined from uniaxial load tests. For bi-axial stress states, the yielding is identified using yield criteria such as Von-Mises, 
Tresca etc. Incremental finite element analysis is carried out for this problem. The most useful finite element formulation is presented as the 'initial stress' method by Zienkiewicz et al (1969).

\subsection{Contact stress analysis}

There has been extensive literature on contact stress analysis in problems involving changing contact between two elastic bodies with load level. Here the stress and displacement fields are nonlinear with applied load due to the changing configuration. In the presence of elasto-plastic deformation, there would be two types of nonlinearities occurring together. The literature dealing with the analysis of problems with both these nonlinearities is scanty. The only attempt in this direction seems to be by Brombolich (1973) who analysed the fastener joint problem combining the two nonlinearities but the details of his work are not available. This can be attributed to the fact that these problems are computationally intensive. It is possible to attempt these problems now, due to the present day availability of large computing power, though tackling them would have been only a dream several years ago.

\subsection{Numerical methods for estimation of crack closure levels}

Two-dimensional elasto-plastic finite element analysis of crack growth and closure has been conducted by several investigators over the years. Most of this work was extensively reviewed by Seshadri (1995). Newman and coworkers were one of the first to numerically study crack closure phenomenon in centre-cracked panels under constant amplitude loading or two-level block loading (Newman \& Armen 1975; Newman 1976). Their numerical estimates of crack closure were qualitatively consistent with experimental measurements. Another numerical simulation of crack closure was by Ogura \& Ojhi (1977) who studied crack growth from notches under variable amplitude loading. Special crack tip elements and translation of near crack tip mesh was used by Nakagaki \& Atluri (1980). Blom \& Holm (1985) compared numerical estimates of crack closure in CT specimens at different stress ratios with experimental measurements. Lalor \& Sehitoglu (1988) contributed extensively to numerical study of the crack closure phenomenon from notches under constant amplitude loading.

Later on McClung \& Sehitoglu (1989) made an extensive review of the basic modelling issues and came out with certain criteria which had to be met in the finite element model. One of the most important criteria was the refinement of the mesh along the crack line. This will be discussed later in the numerical results. Another important issue is the node release scheme to simulate fatigue crack growth. The most common scheme is that suggested by Newman \& Armen (1975) which releases the crack tip node at the maximum load in each cycle. Newman completed redistribution of the load due to node release before proceeding with the analysis. A similar technique was employed by Chermahini et al (1988) and Blom \& Holm (1985). Ogura \& Ojhi (1977) released the crack tip at the minimum load in the cycle. Nakagaki \& Atluri (1980) released the crack tip load at different points along the forward loading excursion and found that the opening level was dependent on the timing of the node release. Lalor \& Sehitoglu (1988) employed a node release scheme in which 
the crack tip was advanced immediately after the point of maximum load, during every increment of unloading. Three primary node release schemes were considered by McClung \& Sehitoglu (1989). They were, node release at maximum load with redistribution, node release at minimum load and node release immediately after maximum load. Each of these schemes involved release of a node in every cycle. It was shown generally that the variation of results between the different node release schemes is not significant.

\subsection{Elasto-plastic crack tip parameter}

There were serious attempts in the past to propose alternate fracture parameters in the absence of crack closure effects, to fit the crack growth behaviour in the presence of elastoplastic deformations at the crack tip. The work of Rice (1968) led to the development of the well-known $J$-integral concept. Based on the assumption of self-similar crack growth, the $J$-integral was derived. With inelastic deformations, the $J$-integral can still be used as long as there is proportional loading and there is no unloading. However, once crack growth commences, $J$ ceases to be the crack-tip parameter (Brust et al 1985). Again, with incremental flow theory of plasticity, $J$ ceases to be a crack tip parameter as it loses its path independence and the physical interpretation of the same is not valid. However, for stationary cracks, Hutchinson (1968) and Rice \& Rosengren (1968) have shown that $J$ is still a controlling parameter under small scale and fully plastic conditions with power law hardening materials. Also, Hutchinson \& Paris (1979) opined that $J$ is still a crack tip parameter for small amount of crack growth and becomes invalid at higher levels.

Number of alternative crack-tip fracture parameters were tried. Crack-Tip Opening Displacement (CTOD) at the crack tip were monitored and used as an alternative parameter (Wells 1962). Crack-Tip Opening Angle (CTOA) was also another parameter tried for crack-growth correlation. But, it is difficult to accurately determine the value of CTOA through experiments. Usually, a combination of $J$ and CTOA was used in many situations. Again in case of mixed mode loading, CTOA does not describe the crack growth. Strain Energy Release Rate (SERR or $G$ ) was tried as a possible crack-tip parameter (Nakagaki et al 1979).

All the above methods were found to be unsatisfactory. In search of an alternative fracture parameter in the elasto-plastic regime, a new path independent parameter $\left(\Delta T_{c}\right)$ was proposed. This can be used with flow theory of plasticity and can account for loading and unloading and can take any arbitrary loads. This was later modified by Atluri \& Nishioka (1982) and a new parameter $\left(\Delta T_{P}^{*}\right)$ was defined. This is a direct measure of crack-tip field with flow theory of plasticity and under deformation theory of plasticity it is equivalent to $J$ defined by Rice (1968).

\subsection{Application to cyclic loading}

In case of cyclic loading which leads to cyclic plastic deformation, the application of the $J$ integral in its original form is questionable as it loses path independence. It is assumed, however, that crack growth occurs during the loading portion of any particular cycle and any further plastic deformation or damage occurring in the unloading cycle is reflected in the next subsequent loading cycle. Here, $J$-integral has been redefined with the above 
assumption and the values in the integrand are calculated for only the loading portion of any particular cycle.

Dowling (1977) proposed extension of the concept of $J$-integral to cyclic loading. They carried out fatigue experiments on Compact Tension Specimens (CTS). The $\Delta J$ values were calculated using the area under the curve of the load-deffection curves only during the loading part of any particular load cycle. They had also corrected for the crack closure using only the portion of the area under load deflection curve where the crack is fully open. Here the elasto-plastic data described by $J$-integral were superimposed on the elastic data based on LEFM and a small scatter band was obtained on comparing the results. Dowling (1977) developed equations for calculating the $\Delta J$ using the finite element results of Shih \& Hutchinson (1976) for centre-cracked and edge-cracked panels.

However, it is obvious from literature that the application of $\Delta J$ has various limitations. The present work is derived basically on the concept of incremental flow theory of plasticity, initial stress method and involves cyclic loading. Hence, $\Delta T_{P}^{*}$ proposed by Atluri \& Nishioka (1982) to characterize stable crack growth in elasto-plastic regime is used. This parameter can be used for arbitrary loading, unloading and with incremental flow theory of plasticity.

\section{Finite element analysis}

The finite element analysis adopted is an incremental elasto-plastic analysis under cyclic loading, combined with contact stress problems in certain ranges of loading. The formulations were carried out in three-dimensions and this could easily be reframed to study problems in two-dimensions. The main feature of these problems is the two types of nonlinearity arising out of varying contact and material behaviour. This has been dealt with by a novel approach using a marching solution. The basic FEM formulation is straight-forward and will not be dealt with in detail. Three-dimensional analysis is carried out using 8noded iso-parametric brick elements and two-dimensional analysis with four-/eight-noded quadrilateral elements. The Von Mises yield criterion is:

$$
\left.\begin{array}{rl}
F\left(\sigma_{i j}, \alpha_{i j}\right)=[ & \left(\sigma_{x}-\alpha_{x}\right)^{2}+\left(\sigma_{y}-\alpha_{y}\right)^{2}+\left(\sigma_{z}-\alpha_{z}\right)^{2} \\
& -\left(\sigma_{x}-\alpha_{x}\right)\left(\sigma_{y}-\alpha_{y}\right)-\left(\sigma_{y}-\alpha_{y}\right)\left(\sigma_{z}-\alpha_{z}\right) \\
& -\left(\sigma_{z}-\alpha_{z}\right)\left(\sigma_{x}-\alpha_{x}\right)+3\left\{\left(\tau_{x y}-\alpha_{x y}\right)^{2}\right. \\
& \left.\left.+\left(\tau_{y z}-\alpha_{y z}\right)^{2}+\left(\tau_{z x}-\alpha_{z x}\right)^{2}\right\}\right]^{\frac{1}{2}}-\overline{\sigma_{y}},
\end{array}\right\}
$$

where $\sigma_{x}, \sigma_{y}, \sigma_{z}, \tau_{x y}, \tau_{y z}, \tau_{z x}$ are the stresses corresponding to the current state; $\alpha_{x}, \alpha_{y}$, $\alpha_{z}, \alpha_{x y}, \alpha_{y z}, \alpha_{z x}$ are the components of the back stresses, representing the translation of the centre of the yield surface, and $\overline{\sigma_{y}}$ is the current yield stress.

If the stress state is such that, $F\left(\sigma_{i j}, \alpha_{i j}\right)<0$, the material is in the elastic state. When $F\left(\sigma_{i j}, \alpha_{i j}\right)=0$, the inelastic state is initiated. Subsequent plastic behaviour under increasing stress and strain is determined by the flow theory of plasticity. The total incremental strain has elastic $\left(\Delta \epsilon_{e l}\right)$ and plastic $\left(\Delta \epsilon_{p l}\right)$ components as follows:

$$
\Delta \epsilon=\Delta \epsilon_{e l}+\Delta \epsilon_{p l} .
$$




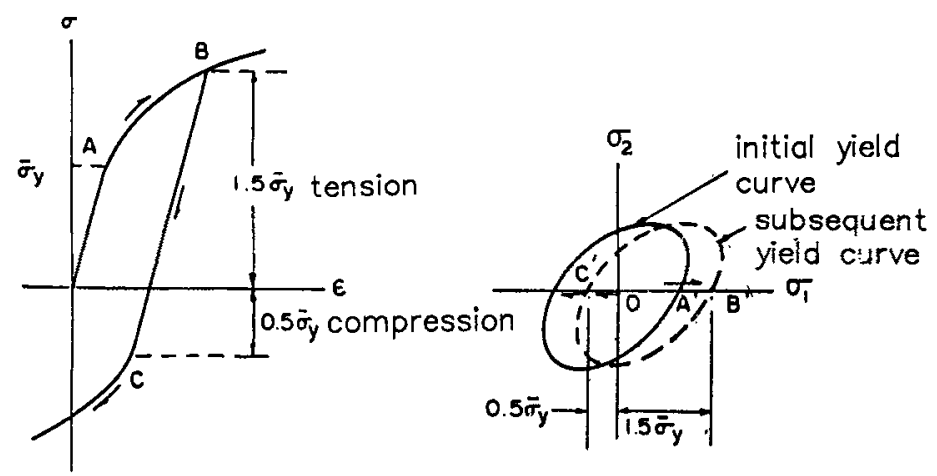

Figure 2. Kinematic hardening.

The incremental plastic strain is obtained using an associated flow rule, based on Drucker's Postulate for work-hardening material. The flow rule can be written as

$$
\Delta \epsilon_{p l}=\mathrm{d} \lambda \frac{\partial F\left(\sigma_{i j}, \alpha_{i j}\right)}{\partial \sigma_{i j}},
$$

where $\mathrm{d} \lambda$ is a positive or a scalar quantity.

When yielding occurs, the stress state must remain on the translated yield surface. This can be derived as follows. By total differentiation of (3) we get,

$$
\mathrm{d} F=\left\{\frac{\partial F}{\partial \sigma}\right\}^{T} \mathrm{~d} \sigma+\left\{\frac{\partial F}{\partial \alpha}\right\}^{T} \mathrm{~d} \alpha
$$

The condition is that $\mathrm{d} F=0$ to remain on the yield surface. Further,

$$
\left\{\frac{\partial F}{\partial \sigma_{i j}}\right\}^{T}=-\left\{\frac{\partial F}{\partial \alpha_{i j}}\right\}^{T},
$$

since $F$ is a function of $\left(\sigma_{i j}, \alpha_{i j}\right)$. So, (6) can be rewritten as

$$
\left(\partial \sigma_{i j}-\partial \alpha_{i j}\right) \frac{\partial F}{\partial \sigma_{i j}}=0
$$

\subsection{Hardening rule}

The finite element analysis, in particular under cyclic loading covering both loading and unloading portions, should properly represent the hardening portions of the stress-strain curve. At any stage of yielding, the stress state corresponds to an yield surface (or curve in two-dimensions). Once the yield surface at a stress state is known, the next issue will be what is the shape, size and position of the subsequent yield surface?

A power law uniaxial stress-strain response (Ramberg-Osgood type) in a material is shown in figure 2. At a particular stage let $B$ be the position on the stress-strain curve during the loading portion. For most of the practical materials it has been observed that increase in the yield limit in tension is accompanied by a reduction in yield limit in compression. The yielding in compression is represented by $\mathrm{C}$ resulting in anisotropic 
hardening (obviously, the case of isotropic hardening corresponds to that where an increase in yield limit in tension is accompanied by an equal increase in yield limit in compression). This anisotropic hardening is known as kinematic hardening and the phenomenon of stress induced anisotropy is known as the Bauschinger effect.

The kinematic hardening results in translation of the yield surface. A simple case of translation of yield surface is shown in figure 2 . In problems involving plastic deformations during loading and reversed plasticity during unloading, it is essential to use the realistic kinematic hardening. In the present cases, the hardening rule proposed by Prager (1955) and later modified by Ziegler (1967) is used in the evaluation of the back stresses which represent the coordinates of the yield surface

$$
\mathrm{d} \alpha_{i j}=\mathrm{d} \mu\left(\sigma_{i j}-\alpha_{i j}\right),
$$

where the scalar $\mathrm{d} \mu$ ensures the condition that the stress state must remain on the translated yield surface. On substitution of (9) into (8), it is possible to derive that

$$
\mathrm{d} \mu=\left(\partial F / \partial \sigma_{i j}\right) \mathrm{d} \sigma_{i j} /\left[\left(\sigma_{i j}-\alpha_{i j}\right)\left(\partial F / \partial \alpha_{i j}\right)\right] .
$$

'The equation of equilibrium which needs to be solved can be written as,

$$
\left[K_{e}\right]\{U\}=\{P\}+\{Q\},
$$

where $\{P\}$ is the applied force vector, $\{Q\}=\int B^{T} \Delta \sigma^{\prime \prime} \mathrm{d} V,\left\{\Delta \sigma^{\prime \prime}\right\}=\left\{\Delta \sigma^{\prime}\right\}-\{\Delta \sigma\}$, $\left\{\Delta \sigma^{\prime}\right\}=$ elastic increment of stress for given $\mathrm{d} \epsilon,\{\Delta \sigma\}=$ true increment of stress for given $\mathrm{d} \epsilon$.

While carrying out the analysis for plane strain condition it is necessary to avoid plane strain locking in the formulation.For this purpose the total strain energy is divided as,

$$
U_{\text {total }}=\frac{1}{2} G \int_{v} \epsilon_{d}^{T} D \epsilon_{d} \mathrm{~d} v+\frac{1}{2} \lambda \int_{v} \epsilon_{v}^{T} \epsilon_{v} \mathrm{~d} v
$$

where $\epsilon_{d}$ is the distortional strain and $\epsilon_{v}$ is the volumetric strain. $G=E / 2(1+v)$, $K=E / 3(1-2 \nu)$ and $\lambda=(K-2 G / 3)$. The locking was overcome by reduced integration employed for evaluating volumetric energy which makes the dilatational strains constant within the element (Satish Chander \& Prathap 1989).

\subsection{Contact stress analysis}

As observed earlier, several practical problems have changing contact situations combined with material nonlinear behaviour. Typical examples of these cases in numerical evaluation will be presented in this paper.

Contact between two elastic bodies is represented by bounding conditions termed as ambiguous. Consider for example the problem of contact between two bodies $A$ and $B$ as in figure 3. Let $C_{A}$ and $C_{B}$ represent the regions in $\mathrm{A}$ and $\mathrm{B}$ respectively which could be in contact under certain load distribution. The contact is assumed to be frictionless so that nodes 1 and 2 in $C_{A}$ and $C_{B}$ respectively could establish contact with each other and are free to slide. The boundary conditions on the interface could be written as, 

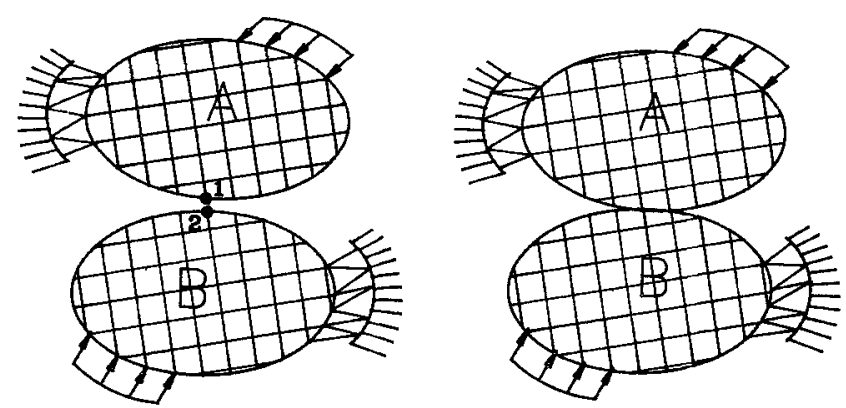

Figure 3. A typical contact stress problem.

Case I : Nodes 1 and 2 are in contact.

$$
\begin{aligned}
F_{t 1}= & F_{t 2}=0, U_{n 1}=U_{n 2} \\
& F_{n 1}+F_{n 2}=0 \\
& \text { and } F_{n 2} \leq 0
\end{aligned}
$$

so that the contact surface has compressive load.

Case II: Nodes 1 and 2 are not in contact.

$$
\begin{gathered}
F_{t 1}=F_{t 2}=0, F_{n 1}=F_{n 2}=0 \\
\text { and } U_{n 2} \leq\left(U_{n 1}+\Delta\right),
\end{gathered}
$$

where $n$ and $t$ refer to directions normal and tangential to the contact surfaces, $F$ and $U$ represent forces and displacements respectively, and $\Delta$ is the appropriate initial gap between the points.

The inequality constraints are ambiguous and they ensure that the normal stress at the contact surface is compressive and displacements in the region of no contact are not overlapping.

When there is a possibility of changing extents of contact/separation between the two bodies, there would be a moving boundary value problem. A node could be either in the region of contact/separation depending on the load level. The stress and displacement fields are nonlinear with load level.

\subsection{Cyclic loading : Incremental-iterative solution}

The software developed combining elasto-plastic and contact-stress analysis is described in a block diagram (figure 4). The software can handle both loading and unloading by carefully monitoring the two types of nonlinearities.

The solution is incremental-iterative in nature and primarily uses force/displacement extrapolation for advancing/receding contact as a part of marching solution. The force extrapolation for the case of receding contact is described below.

The solution takes advantage of the discrete character of finite element solution. In the absence of inelastic deformation, the solution is linear in the range of loading when contact extends from one node to the neighbouring node. In such a case, solution can be extrapolated linearly so that the forces at the end of contact region are equal to zero 


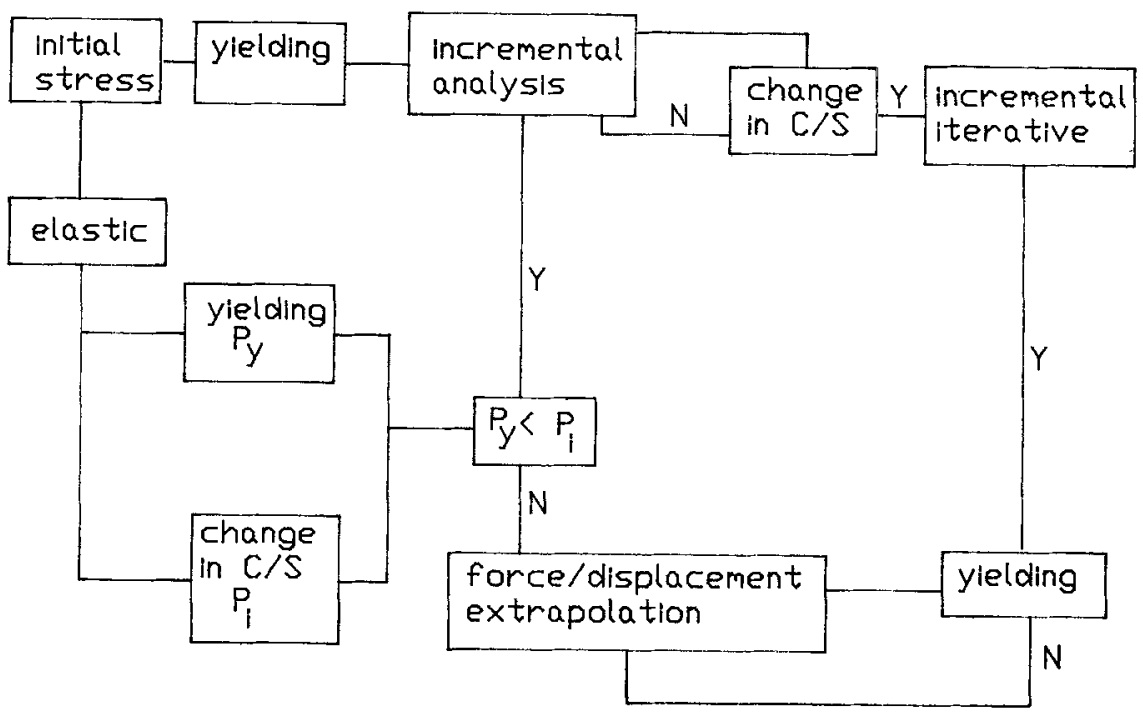

Figure 4. Block diagram for elasto-plastic contact stress analysis.

(figure 5). In the presence of inelastic deformation a similar approach is followed. Here, the solution has only material nonlinearity (and no contact variation) when contact extends from one node to the neighbouring node. Hence, a marching solution is carried out for the contact progressing from node to node, and a purely material nonlinear analysis is conducted between the progress of contact from node to node. A considerable amount of computational time is saved using this approach to deal with the two different types of nonlinearities.

\subsection{Elasto-plastic fracture parameter, $\Delta T_{p}^{*}$}

In the absence of crack closure, the elasto-plastic fracture parameter $\Delta T_{p}^{*}$ (Atluri \& Nishioka 1982) was used in the present work to correlate fatigue crack growth data. This path-independent integral could be evaluated as (figure 6).

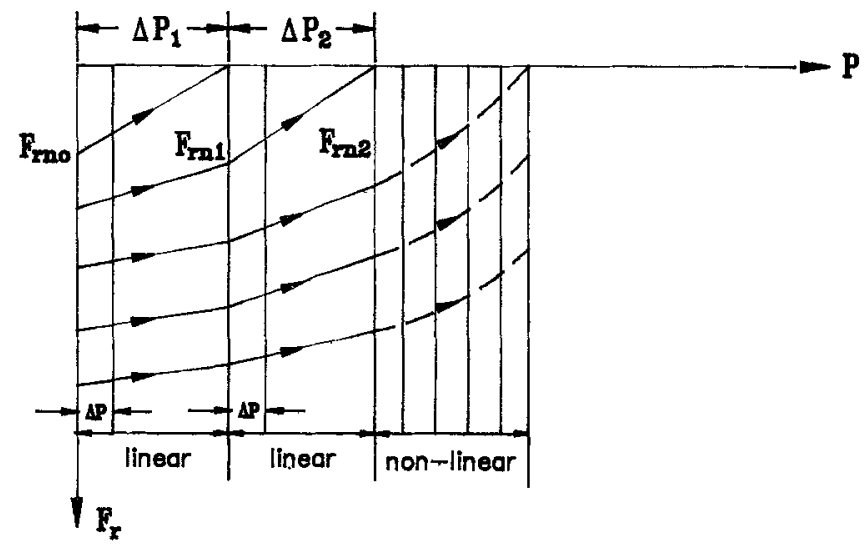

Figure 5. Schematic variation of radial forces with applied load. 


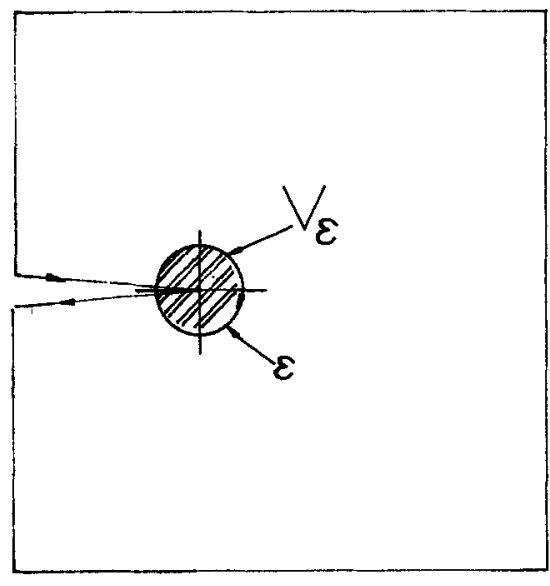

Figure 6. Evaluation of $\Delta T_{p}^{*}$.

$$
\Delta T_{p}^{*}=\int_{\Gamma_{\epsilon}}\left[\Delta \overline{W_{n, 1}}-\left(t_{i}-\Delta t_{i}\right) \Delta u_{i, 1}-\Delta t_{i} u_{i, 1}\right] \mathrm{d} s
$$

where $\Delta \vec{W}=\left(\sigma_{i j}+\frac{1}{2} \Delta \sigma_{i j}\right) \Delta \epsilon_{i j, 1}, t_{i}=\sigma_{i j} n_{j}$, and $\bar{W}$ is the strain energy density, $t_{i}$ is the traction vector, $(, 1)$ refers to the derivatives $\partial / \partial u$. This parameter can be evaluated along a contour $\Gamma_{\epsilon}$ very close to the crack tip.

\section{Numerical results}

Numerical studies are conducted on two different problems when elasto-plastic contact stress analysis plays a significant role. In the first, FE estimate of crack closure stresses is carried out in CTS specimen under two blocks of loading of Lo-Hi and Hi-Lo sequence. The second problem is the case of lug joint with rigid interference pin and two diametrically opposite cracks at the hole boundary normal to the direction of loading.

\subsection{Crack closure estimation}

Numerical studies are conducted on a compact tension specimen shown in figure 7 . The width of the specimen is $W=40 \mathrm{~mm}$. The specimen is loaded by two pins as shown. The
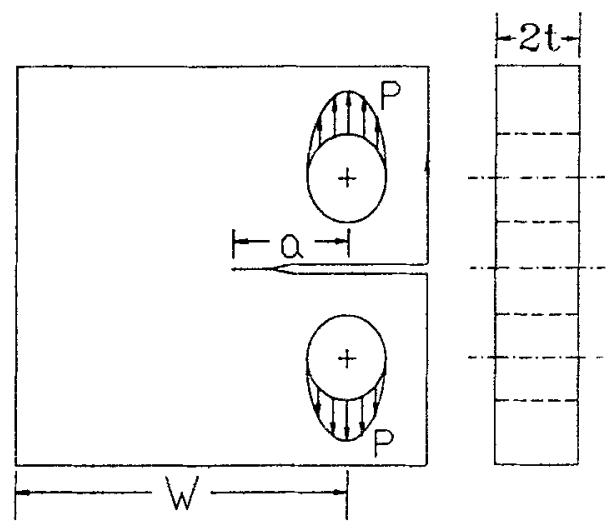

Figure 7. Loading on compact tension specimen. 


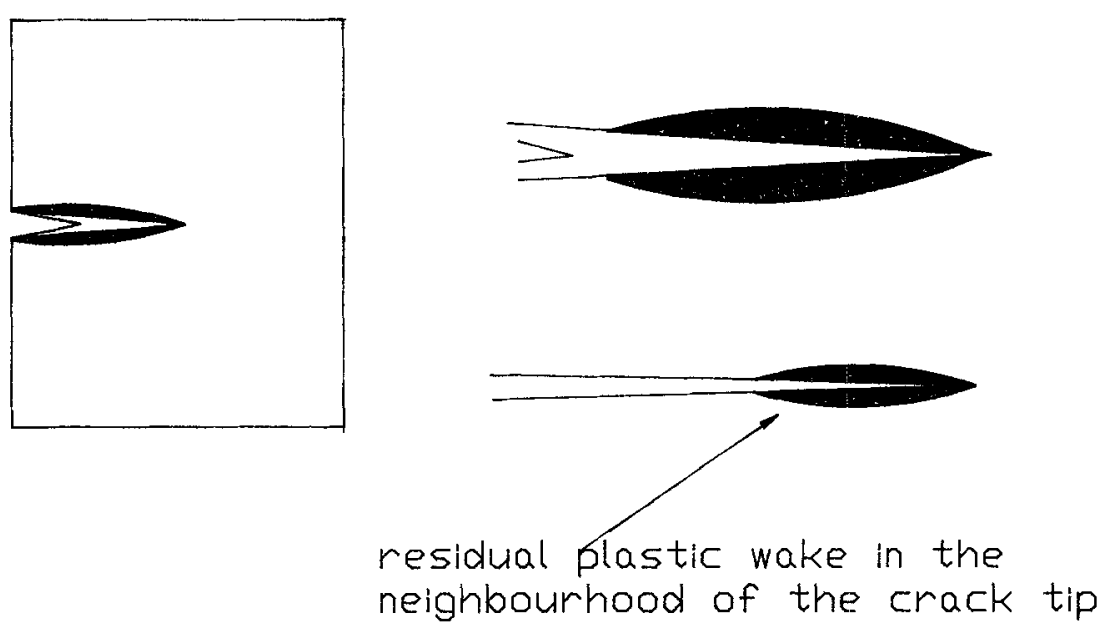

Figure 8. Development of plastic wake.

distance between the crack tip and the load line is denoted as crack length ' $a$ '. The loading is idealized as acting on half of the hole boundary in the form of cosine distribution. The equilibrium is given by

$$
2 \int_{0}^{\pi / 2} \sigma_{r} r \cos \theta \mathrm{d} \theta=P
$$

where $r$ is the radius of the hole boundary.

There are a few issues to be considered in finite element analysis under cyclic loading. (1) The first and the foremost is the crack-tip mesh refinement. This has been examined in the literature earlier (Newman 1976; McClung \& Sehitoglu 1989). It is generally found that the mesh should be fine enough so that, when the crack is advanced after every cycle, it simulates real crack growth. On the other hand, if it is made too fine, the computational requirements become too large. One has to achieve a balance between these two requirements. The aim of the exercise is to accurately estimate crack-closure stress and it is seen that this estimation is not improved in its accuracy over certain levels of mesh refinement. Hence, in the current study, the crack-tip element size was chosen, after certain numerical experiments, as 25 microns. (2) The crack-tip advance scheme is the next important issue. It was discussed in the introduction that several investigators earlier used different schemes and ultimately it was found by McClung \& Sehitoglu (1989) that the difference between these two schemes is small. In the present study, the crack-tip node release scheme suggested by Newman (1976) is adapted. Here, the node is released at the maximum stress in each cycle and the resulting unbalanced stresses are redistributed. (3) As the crack advances, it leaves a residual plastic wake behind. Finite element simulation of this phenomenon for all the fatigue cycles would be computationally impossible. Here, it is postulated that when the crack is at a particular length the crack-closure stress estimates are only influenced by the residual plastic wake in the immediate neighbourhood behind the crack tip. This is shown in figure 8. For this purpose the crack is grown over 10 to 15 cycles to simulate the residual plastic wake behind, before the crack-closure stress is estimated at any crack length. Numerical studies showed that this provides sufficiently accurate values for crack-closure stresses. (4) Crack closure is identified as the load at 

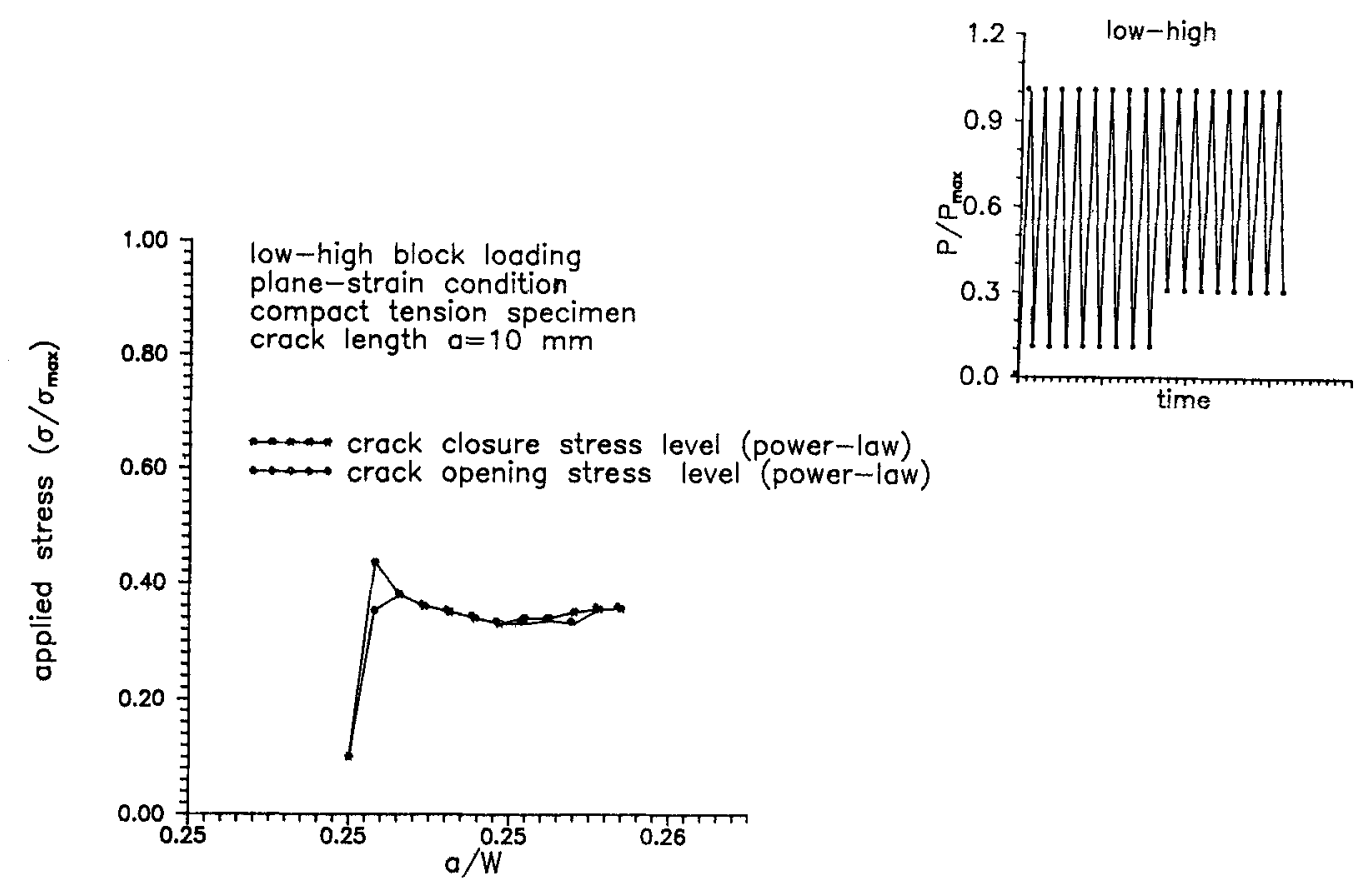

Figure 9. Normalised crack closure and opening in low-high block of loading.

which the first pair of nodes behind the crack tip touch each other during the unloading part of the cycle.

The compact tension specimen is subjected to Hi-Lo and Lo-Hi blocks of loading. The high block of loading corresponds to $R=0.3\left(\sigma_{\min } / \sigma_{\max }=0.3, \sigma_{\min }=0.3\right)$. The low block of loading corresponds to $R=0.1\left(\sigma_{\min } / \sigma_{\max }=0.1, \sigma_{\min }=0.1\right)$. In the high block of loading the crack-closure stress has stabilised to a value $\sigma_{c l} \approx 0.35$, and in the low block of loading $\sigma_{c l} \approx 0.33$ (figures 9 and 10). This is the influence (though minor) of the stress ratio on the crack-closure stress. The values compare with those reported before by Seshadri (1995). In between high-low or low-high blocks of loading, the crack-closure stress transits between these two values.

\subsection{Preliminary studies on $\Delta T_{p}^{*}$ integral}

Certain preliminary studies were conducted (Satish Kumar et al 1994) on the path independent integral $\Delta T^{*}$ and compared with results in literature. The analysis was conducted on CTS specimen shown in figure 11. Crack length of $26.4 \mathrm{~mm}$ was used in the analysis. The material properties and the geometry of the specimen are same as that used by Atluri \& Nishioka (1982).

The incremental load was applied as incremental displacements and the load-deflection curve obtained from this analysis which is shown in figure 12 , along with the comparative values from the earlier work. The results are in good agreement. $\Delta T_{p}^{*}$ are evaluated for each increment of displacement and the summation is carried out as $T_{p}^{*}=\Sigma \Delta T_{p}^{*}$. Results from the present analysis and those from the literature are shown in figure 13, for different increments of displacement. The study was carried out for the loading portion of a cycle 

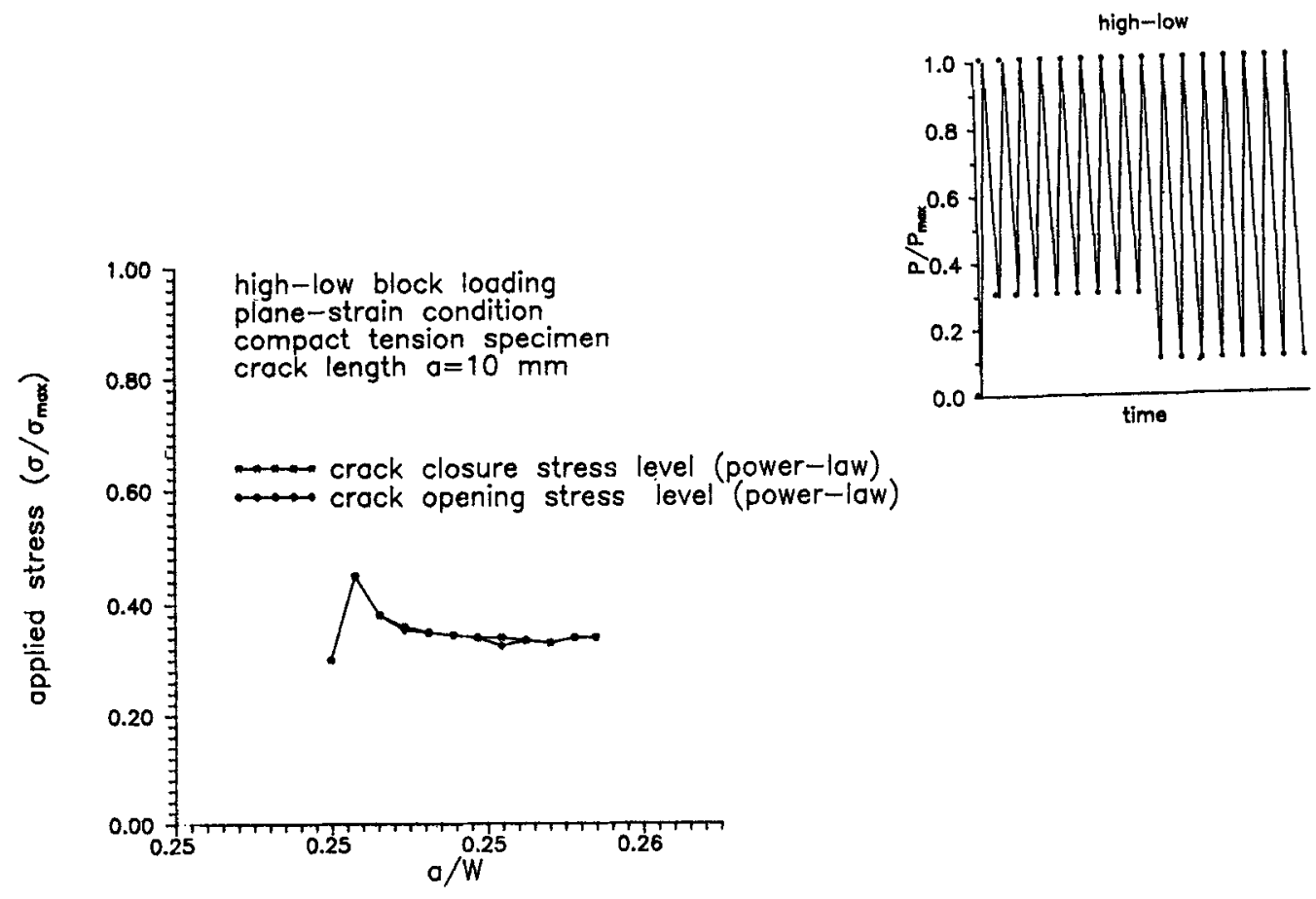

Figure 10. Normalised crack closure and opening in high-low block of loading.

and for a part of the unloading portion. The results compare very well and validate the present software.

\subsection{Cracks around lug joints}

The study was later conducted on a cracked lug joint for a configuration for which certain experimental results were available. The lug was made of HE-15 AST Al-Cu alloy. The geometry of the specimen is shown in figure 14. The lug was fitted with a 4340 steel interference pin. For numerical study, a steel pin of different interference values was chosen. The diameter of the hole in the lug was $2 a$, and the diameter of the pin was $2 a(1+\lambda)$, so that $\lambda$ represents relative interference between the pin and the hole. Numerical study was conducted with $\lambda=0.5,0.75$ and $1.0 \%$.

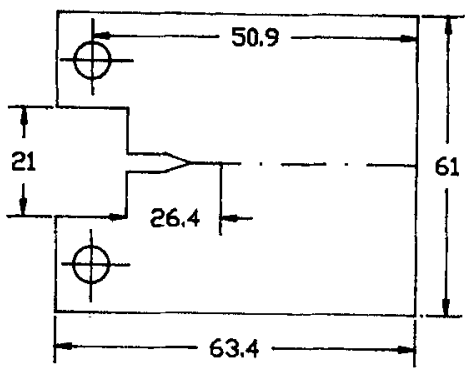

all dimensions are in $\mathrm{mm}$
Figure 11. Dimensions of compact tension specimen. 


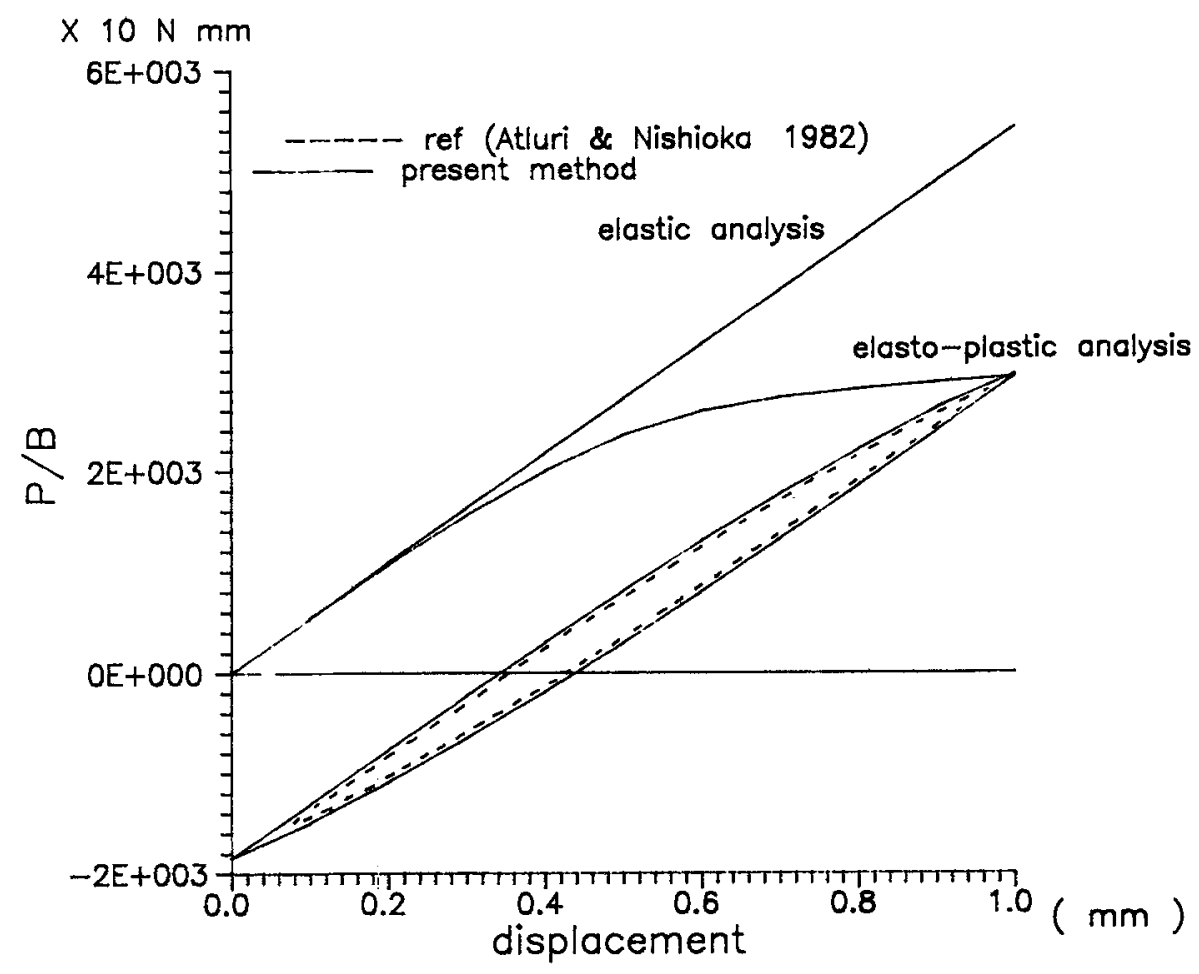

Figure 12. Variation of forces with displacement.

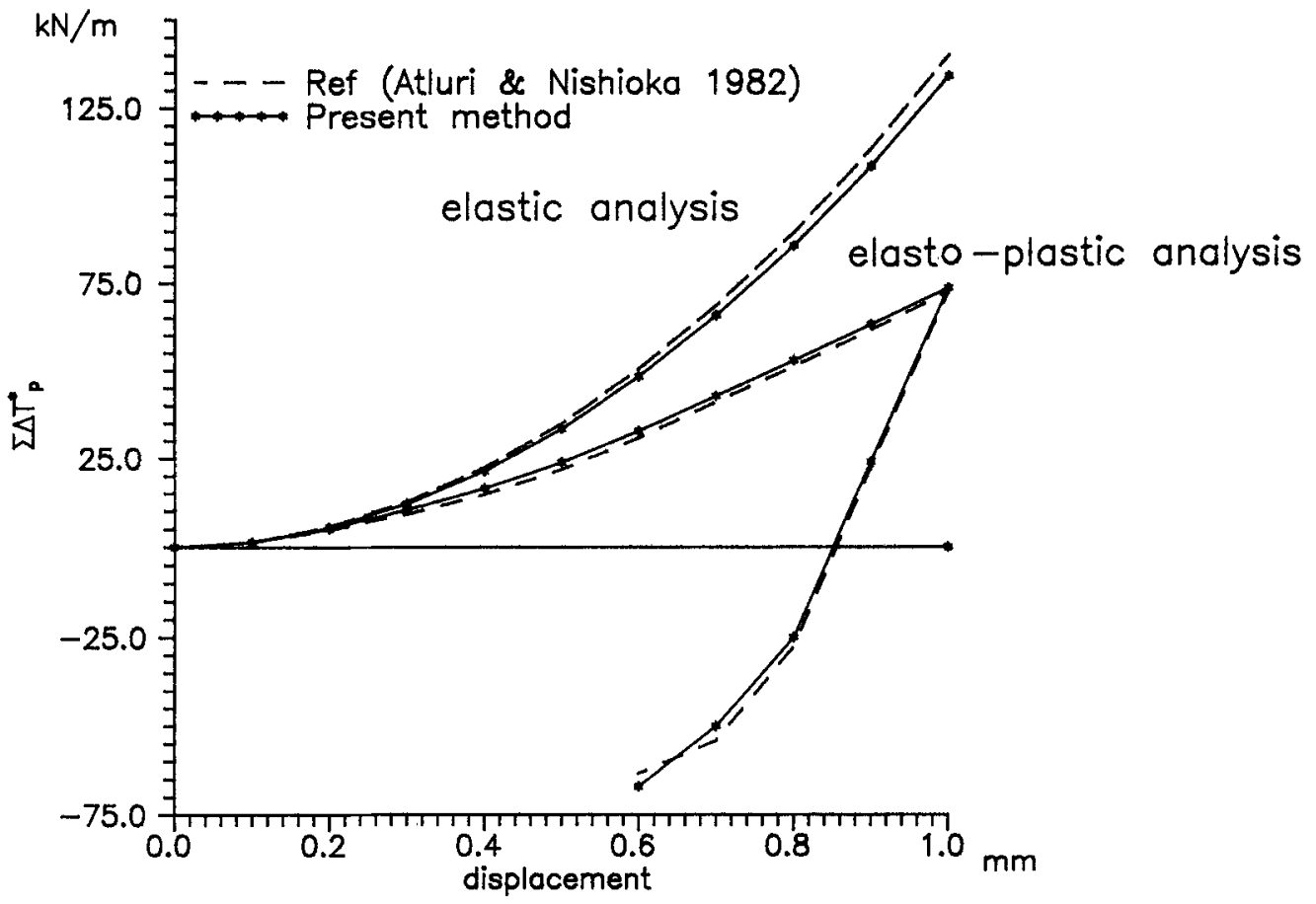

Figure 13. Variation of $\Sigma \Delta T_{p}^{*}$ with displacement. 


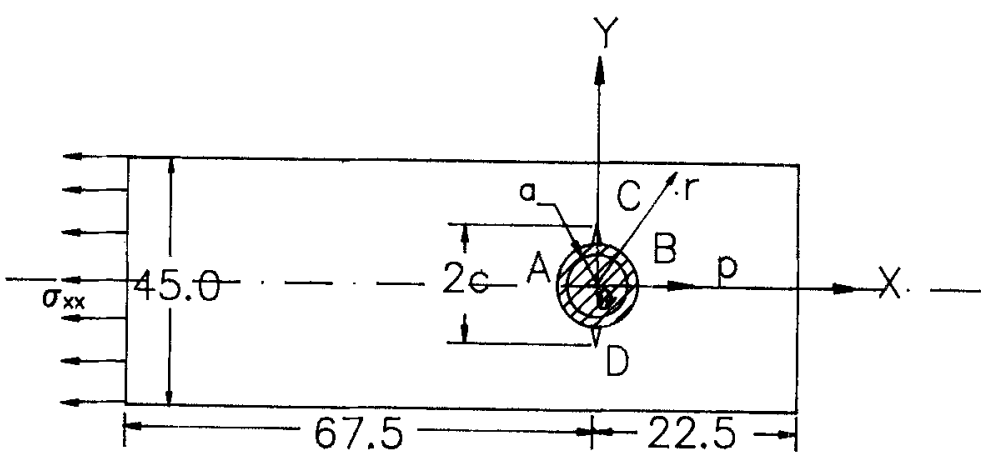

Figure 14. Lug joint with geometrical parameters.

$\Delta T_{p}^{*}$ was computed for different contours around the crack tip. The path independence of this contour integral for two values of interference is shown in figure 15. It is now proposed to use this integral for correlation of fatigue-crack growth data. For this purpose the variation of $\Delta T_{p}^{*}$ vs crack length is shown for a value of $\lambda=0.75 \%$ in figure 16 .

A power law variation of fatigue-crack growth data with $\Delta T_{p}^{*}$ was attempted for different values of interference. For this purpose, FCG data presented earlier (Satish Kumar et al 1995 ) is utilized. The correlation of $\mathrm{d} a / \mathrm{d} N$ (crack growth per cycle) with $\Delta T_{p}^{*}$ on a logarithmic scale is shown in figure 17. The fit is very similar to the Paris equation.

\section{Concluding remarks}

The role of elasto-plastic analysis under cyclic loading in fatigue crack growth studies is briefly reviewed. Finite element analysis dealing with two nonlinearities due to mate-

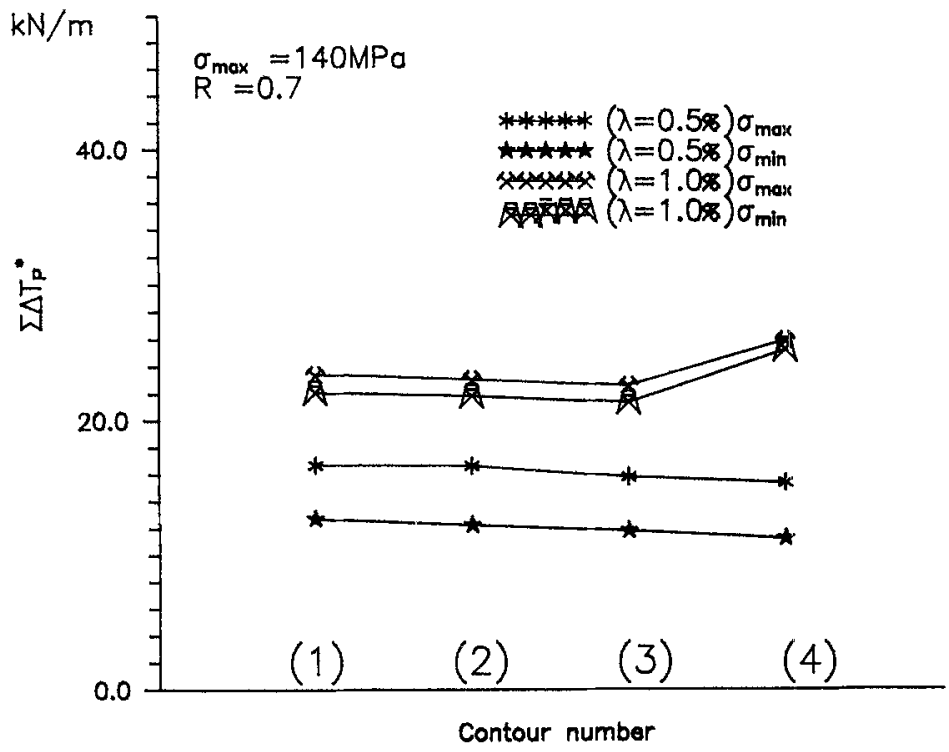

Figure 15. Path independence of $\Sigma \Delta T_{p}^{*}$ integral. 


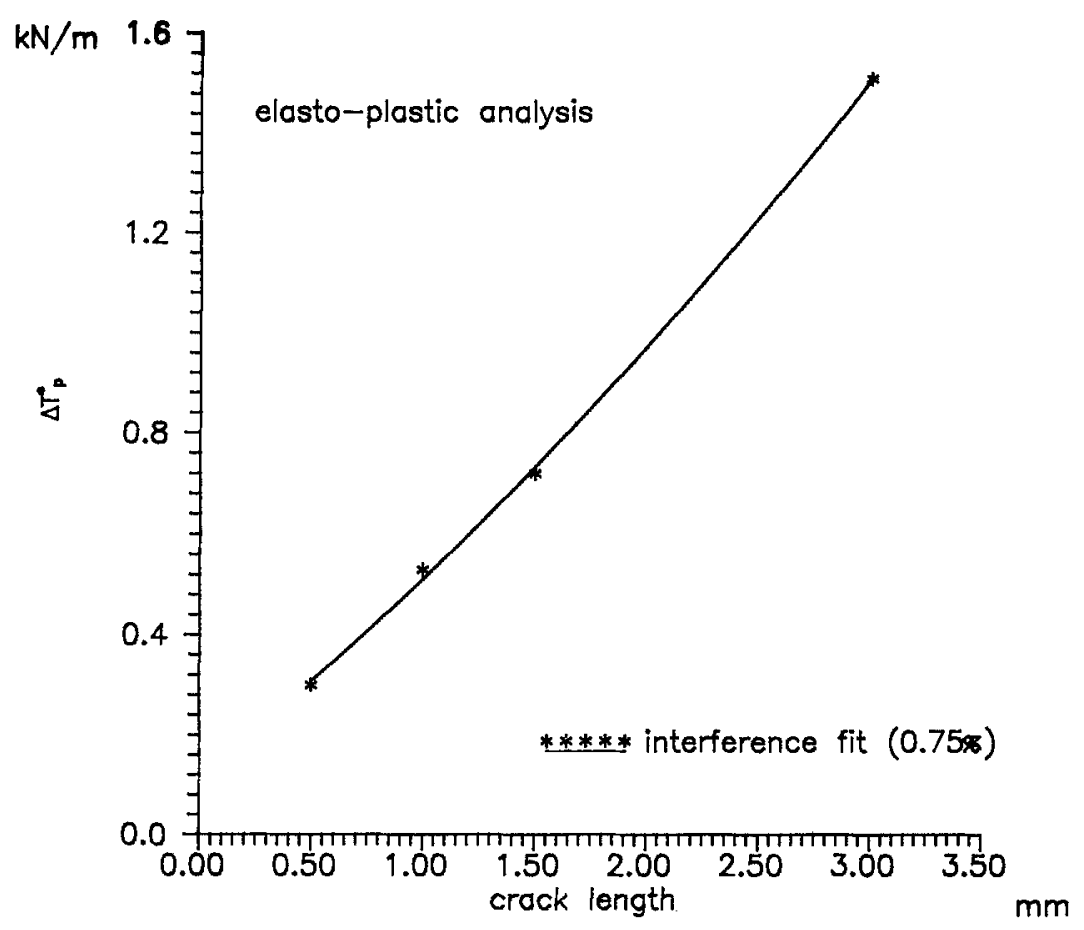

Figure 16. Variation of $\Delta T_{p}^{*}$ with crack length.

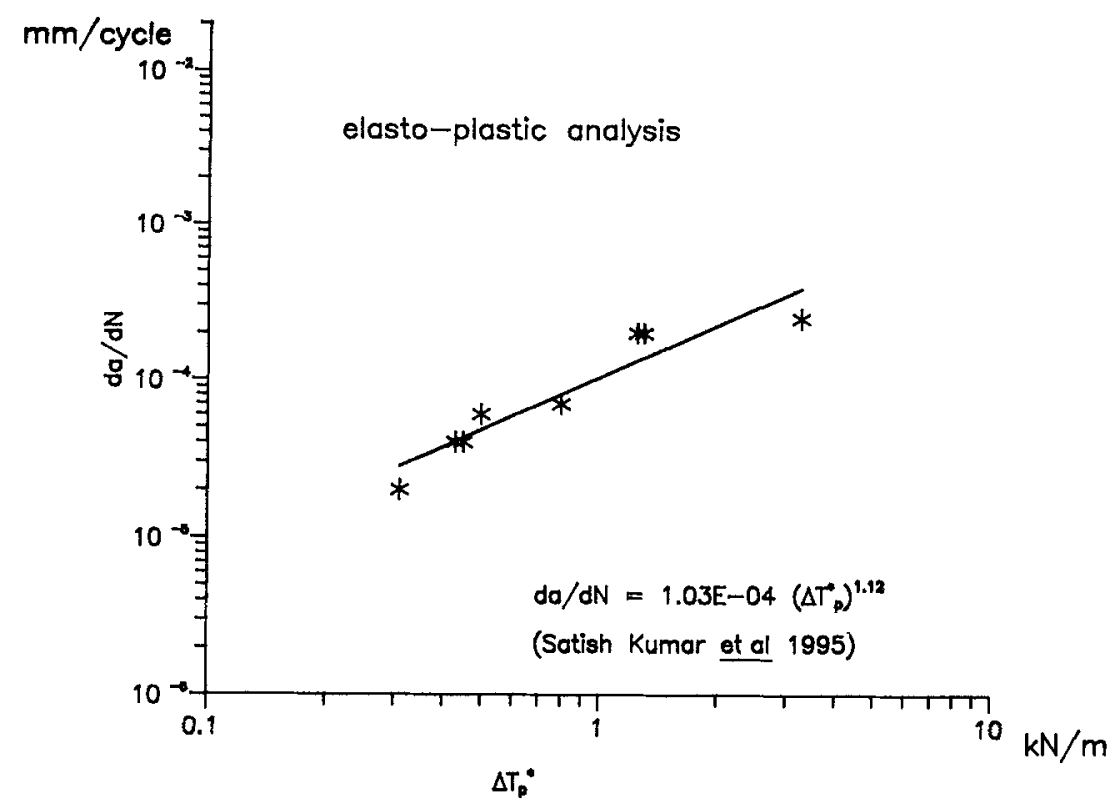

Figure 17. Crack growth rate variation with $\Delta T_{p}^{*}$. 
rial nonlinearity and contact stress analysis in typical problems is presented. Numerical estimates of crack closure stresses in CTS specimens under Hi-Lo and Lo-Hi blocks of loading are presented using the methods and procedures developed. In the absence of crack closure, fatigue-crack growth in structural components is correlated to elasto-plastic fracture parameter $\Delta T_{p}^{*}$ proposed earlier in literature. Correlation of fatigue-crack growth data with this path-independent integral is presented.

The work presented in this paper is supported by sponsored research projects of the Aeronautics R \& D Board, Government of India and their support is acknowledged. The author expresses his thanks to his colleague Prof T S Ramamurthy and his students Dr B R Seshadri, Dr K Satish Kumar and Dr K S Venkatesh for their contributions in this research work.

\section{References}

Atluri S N, Nishioka T 11982 Incremental path-independent integrals in inelastic and dynamic fracture mechanics. Eng. Fract. Mech. 10: 209-244

Blom A F, Holm D K 1985 An experimental and numerical study of crack closure. Eng. Fract. Mech. 22: 997-1011

Broek D 1978 Elementary engineering fracture mechanics, (Alphen aan den Rijn: Sijthoff and Noordhoff)

Brombolich L J 1973 Elastic-plastic analysis of stresses near fastener holes. AIAA paper 73-252, 11th Aerospace Sciences meeting, Washington DC

Brust F W, Nishioka T, Atluri S N 1985 Further studies on elasto-plastic stable fracture utilizing the $T^{*}$ integral. Eng. Fract. Mech. 22: 1079-1103

Chermahini R G, Shivakumar KN, Newman JC 1988 Three-dimensional finite element simulation of fatigue crack growth and closure. ASTM STP 982, pp 398-413

Dowling N E 1977 Crack growth during low-cycle fatigue of smooth axial specimens. ASTM STP 637, pp 97-121

Elber W 1970 Fatigue crack closure under cyclic tension. Eng. Fract. Mech. 2: 37-45

Forman R G, Kermey V E, Engle R M 1967 Numerical analysis of crack propagation under cyclic loaded structures. J. Basic Eng. 89: 459-464

Hutchinson J W 1968 Singularity behavior at the end of a tensile crack in a hardening material. J. Mech. Phys. Solids 16: 13-31

Hutchinson $J$ W, Paris P C 1979 Stability analysis of $J$-controlled crack growth. ASTM STP 668, pp 37-64

Lalor P L, Sehitoglu H 1988 Fatigue crack closure outside small scale yielding regime. ASTM STP 982, pp 342-360

McClung R C, Sehitoglu H 1989 On the finite element analysis of fatigue crack closure-1, basic modeling issues -2, Numerical results. J. Eng. Fract. Mech. 33: 237-272

Nakagaki M, Atluri S N 1980 Elastic-plastic analysis of fatigue crack closure in Mode I and II. AIAA J. 18: 1110-1117

Nakagaki M, Chan W H, Atluri S N 1979 A finite element analysis of stable crack growth. ASTM STP 668, pp 195-213 
Newman J C Jr 1976 A finite element analysis of fatigue crack closure. ASTM STP 590, pp 281-301

Newman J C Jr, Armen H Jr 1975 Elasto-plastic analysis of a propagating crack under cyclic loading. AIAA J. 13: 1017-1023

Ogura K, Ojhi K 1977 FEM analysis of crack closure and delay effects in fatigue crack growth under variable amplitude loading. Eng. Fract. Mech. 9: 471-480

Paris P C, Gomez M P, Anderson W E 1961 Trends Eng. 13: 9-14

Prager W 1955 The theory of plasticity. A survey of recent achievements. Proc. Inst. Mech. Eng. 169: 41

Rice J R 1968 A path independent integral and the approximate analysis of strain concentration by notches and cracks. J. Appl. Mech. 35: 379-386

Rice J R, Rosengren G F 1968 Plane strain deformation near a crack tip in a power law hardening material. J. Mech. Phys. Solids 16: 1-12

Satish Chander, Prathap G 1989 A field consistent formulation for eight-noded solid finite elements. Comput. Struct. 33: 345-355

Satish Kumar K, Dattaguru B, Ramamurthy T S, Raju K N 1994 An algorithm for elastic-plastic contact stress analysis of interference fit pin joints. Int. J. Eng. Anal. Design 2: 71-82

Satish Kumar K, Dattaguru B, Ramamurthy T S, Prakash R V, Raju K N 1995 Presented at 27th National symposium on fatigue and fracture mechanics, Stress intensity estimation for pin loaded lug joints under elastic and inelastic loading. American Society for Testing Materials (ASTM), Williamsburg, Virginia

Seshadri B R 1995 Numerical simulation and experimental correlation of crack closure phenomenon under cyclic loading. Ph D thesis, Indian Institute of Science, Bangalore

Shih CF, Hutchinson J W 1976 Fully plastic solutions and large scale yielding estimates for plane stress crack problems. J. Eng. Mater. Tech. H98: 289-295

Sunder R, Dash P K 1982 Measurement of fatigue crack closure through electron microscopy. Int. J. Fatigue 4: $97-105$

Wells A A 1962 Unstable crack propogation in metals: Damage and fast fracture. Proc of crack propagation symposium (London; College of Aeronautics) 1: 210-230

Ziegler H 1967 A modification of Prager's hardening rule. Q. Appl. Math. 17: 55-65

Zienkiewicz O C, Valliappan S, King I P 1969 Elasto-plastic solutions of engineering problems 'initial stress' finite element approach. Int. J. Numer. Methods in Eng. 1: 75-100 